Página inicial: 427 - Página Final: 440

Tipo de artículo: Investigación

\title{
Configuración del conflicto armado, a partir de la puesta en marcha del Plan Colombia
}

\section{Armed Conflict Setup as of Entry into Operation of Colombia Plan}

\author{
Recibido: abril de 2017 Revisado: mayo de $2017 \quad$ Aceptado:junio de 2017
}

${ }^{1}$ Economista, Universidad Gran Colombia. Experto en tarifas de energía eléctrica, Hidrólogo e Ingeniería Civil. Investigador Corporación Universitaria de Ciencia y Desarrollo UNICIENCIA. Centro de Investigación y Servicio Empresarial. Bucaramanga (Colombia). Contacto: hernando.carrenop@unicienc iabga.edu.co

2 Economista, Universidad Industrial de Santander. Docente de Investigación, Corporación Universitaria de Ciencia y Desarrollo UNICIENCIA. Centro de Investigación y Servicio Empresarial. Bucaramanga (Colombia). Contacto: dianam.garciae@unicienciabg a.edu.co

3 Ingeniero eléctrico, Universidad de los Andes. Master en Ciencias Económicas, Universidad de Pensylvania. Magister en Economía, Universidad de los Andes. Doctorado en Economía. Docente de Investigación, Corporación Universitaria de Ciencia y Desarrollo UNICIENCIA. Centro de Investigación y Servicio Empresarial. Bucaramanga (Colombia). Contato: diegof.oterop@unicienciabga. $\underline{\text { edu.co }}$

\author{
Por: Hernando Carreño Pilonieta ${ }^{1}$, Diana Marcela García Estévez ${ }^{2} \&$ \\ Diego Fernando Otero Prada ${ }^{3}$
}

\begin{abstract}
Resumen
El conflicto armado en Colombia en el Siglo XXI no puede entenderse independientemente del Plan Colombia, en este sentido este acuerdo de cooperación se ha transformado, con sus pros y contras, en elemento central del conflicto en el país, abriendo la puerta a que haya un papel más activo de la comunidad internacional en el mismo. La cooperación militar internacional ha jugado un papel en el conflicto armado interno colombiano
\end{abstract}

Palabras clave. Plan Colombia; relaciones internacionales; conflicto armado; cooperación militar.

\begin{abstract}
The armed conflict in Colombia in the 21 st century cannot be understood independently of the Colombia Plan. In this sense, this cooperation agreement has become, with its pros and cons, a key element of the conflict in the country, opening the door to have a more active role of the international community in it. International military cooperation has played a role in the Colombian internal armed conflict.

Key words: The Colombia Plan; International Relations; Armed Conflict; Military Cooperation.
\end{abstract}




\section{Introducción}

Año 2016, se han cumplido 10 años desde la entrada en vigencia del Plan Colombia, es un momento central para hacer una revisión de su impacto en el conflicto armado interno en Colombia y las transformaciones que a nivel cualitativo dicho conflicto ha tenido en la configuración de los actores armados y no armados del conflicto en el país en los primeros años del siglo XXI.

Este artículo tiene como propósito hacer una revisión de los impactos del Plan Colombia en su fase inicial en el conflicto armado en el país. El planteamiento del eje de investigación se hace a partir de una evaluación de la relación de los actores del conflicto, así como una identificación de los cambios que éste ha tenido como consecuencia de la implementación del Plan. La manera en que se han configurado las relaciones políticas y sociales entre los gobiernos de Estados Unidos y Colombia a partir de la entrada en vigencia del Plan Colombia, principalmente en su primera fase de configuración e implementación que se dio entre 1999 y 2005.

En este sentido, este artículo de análisis se divide en tres partes:

- En la primera de ellas se hará un análisis del surgimiento del Plan Colombia, de los principales actores que jugaron un papel importante en la configuración y puesta en marcha inicial del Plan, así como en los factores determinantes que llevaron a que las relaciones entre Colombia y Estados Unidos tomaran un nuevo rumbo donde la cooperación militar se ubicaría en la centralidad de las relaciones. El Plan Colombia no puede ser entendido de manera independiente a la historia que le precede, por lo cual un elemento transversal en el análisis que aquí se presenta es la revisión de éste desde una perspectiva histórica.

- En la segunda parte se hace una revisión de la fase de ejecución del Plan, específicamente a partir de dos factores de cambio, tanto en Colombia como en los Estados Unidos. El primer cambio a analizar es el viraje en el direccionamiento estratégico para enfrentar el conflicto armado interno en Colombia, el cual como consecuencia del fracaso de los diálogos de paz con la guerrilla de las FARC en San Vicente del Caguán, se dio un giro político hacía una posición donde la estrategia militar primó sobre la del diálogo, al menos en los primeros años del gobierno de Álvaro Uribe Vélez. El segundo factor que es objeto de análisis en esta sección es el ocurrido en Estados Unidos, en primer momento por el cambio de un gobierno demócrata, como el de Bill Clinton que fue promotor del Plan Colombia en su formulación y aprobación, por un gobierno de corte republicano como el de George W. Bush quien asumiría la presidencia desde febrero de 2001 una vez ya había entrado en vigencia el mencionado Plan; asimismo se analiza la manera en que afectó la ejecución del Plan los atentados ocurridos en Estados Unidos el 11 de septiembre de 2001, y la consecuente guerra contra el terrorismo que se desataría como respuesta del gobierno de Estados Unidos y sus aliados militares.

- Finalmente se hará un análisis de la manera en que la entrada en vigencia del Plan afectó a los principales actores del conflicto armado en Colombia, así como los nuevos actores que entraron al escenario con la puesta en marcha de esta ambiciosa estrategia de cooperación militar internacional, la mayor en términos presupuestales que ha tenido Colombia y quizá América Latina. 


\section{El Plan Colombia en perspectiva histórica}

La relación militar entre Estados Unidos y Colombia tiene sus raíces en el siglo XIX, más exactamente en 1848, año en que se firmó el Tratado Mallarino-Bidlack, que fue el instrumento mediante el cual Estados Unidos se encontraba autorizado para realizar intervenciones en Panamá con el fin de sofocar los intentos de separación que allí se presentaban (Mendez, 1976). Fue solamente hasta 1938 que se establecieron relaciones formales de cooperación con el gobierno colombiano; dichas relaciones se fortalecieron con el tiempo.

En 1932, antes del conflicto de Leticia (el conflicto de Leticia se le denomina a la disputa territorial que se dio entre Colombia y Perú que tuvo lugar durante los años 1932 y 1933), llegó a Colombia el primer militar de la fuerza naval de los Estados Unidos, para asistir al país en el diseño de planes para la defensa de los puertos y la compra de armas, dicha asesoría se mantuvo hasta el año 1934 de manera oficial, sin embargo, esta asistencia técnica por parte de un militar norteamericano continuó bajo la figura de consejería privada. Ya para el año de 1938 arribó la primera misión oficial de la fuerza naval de los Estados Unidos a Colombia.

Con el comienzo de las hostilidades en Europa en el año 1939, que dio lugar a la Segunda Guerra Mundial, Estados Unidos reforzó su influencia militar en los países del continente americano. Como señala Russell Ramsey (1981, pág. 99), una misión militar estadounidense llegó a Colombia en 1940 para asesorar en el uso y mantenimiento del equipo militar suministrado dentro de la Ley de Préstamos y Arriendos (Lend Lease Program) y para coordinar una estrategia de defensa común.

Terminada la guerra, la alianza temporal ente la Unión Soviética y Estados Unidos también llegó a su fin, de aliados pasaron a ser rivales. Se da comienzo con ello a la Guerra Fría y, con ella, la guerra por el dominio mundial. Por parte de los norteamericanos se impuso en el decenio de los sesenta lo que se denominó como la Doctrina de la Seguridad Nacional, la cual implicó un cambio en los esquemas de formación de las fuerzas militares y su relación con la sociedad.

A mediados del siglo XX, y como consecuencia de los acuerdos de asistencia militar firmados entre Colombia y los Estados Unidos, el país recibió entre 1961 y 1967 transferencias de recursos por 60 millones de dólares, asimismo como se afirma en Londoño (2011) durante este mismo período le fueron entregados a la nación 430 millones para asistencia económica, los cuales se encontraban en el marco del proyecto internacional de la Alianza para el Progreso.

Así las cosas, la relación entre los dos países tuvo una importante vigorosidad durante la segunda mitad del siglo XX, en el cual el aspecto militar tuvo un rol de trascendencia como tema clave en el desenvolvimiento de las relaciones binacionales. Estas relaciones militares entre los dos países se vieron afectadas en el período de la Guerra Fría, durante el cual, como señala Londoño (2011), hubo un consenso bipartidista entorno a la importancia de mantener como aliado militar al país del norte.

El elemento que permitió la existencia de dicho consenso entre liberales y conservadores fue el establecimiento de un discurso anticomunista, el cual era promovido desde Estados Unidos como estrategia para contrarrestar en las esferas políticas de América Latina el avance del comunismo como alternativa de poder en el continente americano.

En este sentido, cuando emergió el narcotráfico en la región como un lucrativo negocio de economía ilegal, países como Colombia y México estaban alineados con las estrategias militares 
estadounidenses, lo cual facilitó la acción conjunta inicial enfocada en el combate a los carteles del narcotráfico y los cultivos ilícitos del cual se alimentaba este negocio como su principal fuente de abastecimiento de materia prima.

Sería en la década de los años ochenta cuando se iniciaría el proceso de militarización de la guerra contra las drogas, como lo señala Díaz Rivillas (2002). Fue precisamente durante esa década cuando el tema de las drogam empezó a tener un papel cada vezmás relevante en la agenda política y militar de los Estados Unidos, siendo los países del Área Andina losprimero implicados en una estrategia que desde su incio se enfocó principalmente en los productores de drogas,y que así continuaría siendo como se verá más adelante.

\section{Década de los noventa: rompimiento de relaciones}

Ya iniciada la década de los noventa, bajo el gobierno de Ernesto Samper Pizano (1994-1998), se presentó lo que Londoño (2011) denomina como un punto crítico en las relaciones entre Colombia y Estado Unidos, sin embargo, éste no debe considerarse como un punto de quiebre. Es más, desde la perspectiva de este autor se considera que el episodio de deterioro de las relaciones entre los años sirvió como impulsor de la alineación de Colombia con Washington, al punto que se atreve a señalar que existió una profundización de las mismas a partir de allí.

Desde los Estados Unidos se impulsó la tesis de que la falta de cooperación del gobierno de Ernesto Samper contribuyó al crecimiento del narcotráfico en la región, y además generó un ambiente favorable para que la relación de los carteles del narcotráfico y la guerrilla colombiana se estrechara. Para Londoño (2011) esta última situación de estrechamiento de las relaciones entre guerrilla y narcotráfico, tiene varias explicaciones, como la fuerza adquirida por las FARC entre 1996 y 1998, el recrudecimiento del conflicto armado y el aumento de la violencia, que pusieron en entredicho la capacidad de Colombia para combatir a los grupos armados ilegales y mostró la necesidad imperiosa de fortalecer al aparato estatal (Londoño, 2011, pág. 246).

Asimismo, el país como parte de la estrategia de respuesta estadounidense ante el punto crítico por el que atravesaban las relaciones binacionales fue calificado como una narco-democracia en el periodo de gobierno de Samper Pizano, así como un Estado semi-fallido durante el gobierno siguiente encabezado por el presidente Andrés Pastrana (1998-2002).

Ante esta situación en la que se cerraba el panorama de relaciones internacionales para el país, en el cual su mayor socio militar le hacía señalamientos ante la falta de cooperación, se gestó el Plan Colombia como una estrategia de Colombia para dar un paso definitivo en la recuperación de las relaciones con Estado Unidos, y por el lado de Estados Unidos su gran interés era el de tener una mayor presencia militar en la región y controlar el problema de las drogas ilícitas que constituían para el momento la más importante economía ilegal.

Andrés Pastrana, desde el inicio de su período presidencial (1998-2002) declaró la guerra al narcotráfico, por una parte, mientras que su principal bandera de campaña fue la construcción de la paz a través de un proceso de diálogo con la insurgencia. La primera estrategia de guerra contra el narcotráfico, al ser un problema internacional, requería de la alianza y coordinación con otros países, mientras el establecimiento de la paz con los grupos insurgentes hacía necesario instituir un proceso de diálogo que permitiera llegar a un acuerdo con la principal guerrilla, las FARC. 
Gráfico 1. Gasto Militar como porcentaje del PIB (1988-2015)

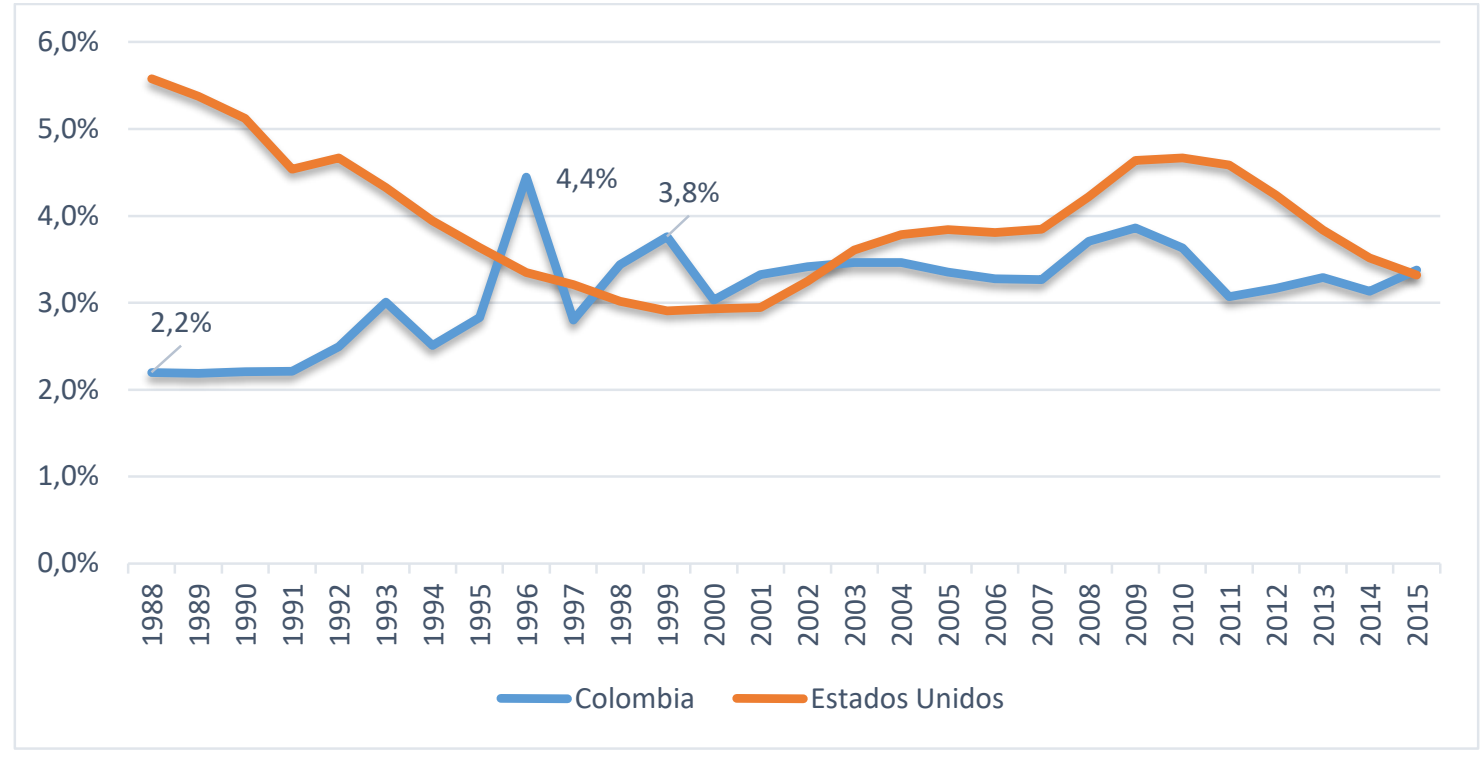

Fuente: Banco Mundial, 2016.

Para realizar una guerra efectiva contra el narcotráfico se hacía necesario un proceso de rearmado y modernización de las fuerzas militares y policiales, lo cual en momentos parecía ir en contra de su principal objetivo como gobierno, la consecución de la paz. ¿Cómo era posible conjugar estas dos estrategias? Por un lado, el proceso de establecimiento de una capacidad armamentista mayor para las fuerzas militares, mientras se hacía una apuesta importante por llegar a un acuerdo de paz con la guerrilla en las mesas de diálogo de San Vicente del Caguán.

La modernización y repotenciación de las fuerzas armadas como apuesta central del gobierno Pastrana requeriría el incremento del gasto militar del país para cubrir las inversiones necesarias para tal fin. Como se observa en el gráfico 1, en el período de 1988 a 1999 se dio un aumento importante del gasto militar como porcentaje del Producto Interno Bruto (PIB), el cual se duplicó en este lapso de tiempo, y se mantendría en la primera década del siglo XXI por encima de la participación en el PIB que se registraba en los primeros años de la década del noventa.

Es posible en este orden señalar que la apuesta por una modernización de las fuerzas militares del país tuvo como respuesta coherente en términos financieros un aumento significativo del gasto militar, que se sumó a las transferencias que se realizaron con ocasión del Plan Colombia. Ahora, de qué manera se posibilitó, por un lado, mantener el discurso de compromiso con la paz al tiempo que se incrementaba el gasto militar. Conjugar y armonizar estos dos elementos, en principio confrontados, constituiría el principal desafío del gobierno Pastrana, y en muchas ocasiones la falta de un discurso coherente alrededor de los dos temas sería uno de los principales obstáculos para el avance de las negociaciones de paz durante este período de finales del siglo XX.

Dentro de la apuesta por la modernización de las fuerzas armadas y su fortalecimiento, el principal jugador internacional era los Estados Unidos, que vio en la formulación de un proyecto de cooperación militar con Colombia una oportunidad de recuperar su presencia en Suramérica. En este sentido se puede asegurar que, a partir de su relación con el proceso de paz que se estaba desarrollando 
en paralelo, el Plan Colombia desde su fase de inicio comenzó siendo un jugador central en lo que sería el desenvolvimiento del conflicto armado en el país en los primeros años del siglo XXI.

Para que surgiera un proyecto de cooperación internacional con una duración de más de una década, en el cual existió un proceso constante de cooperación militar de Estados Unidos hacia Colombia, sólo se pudo dar gracias a la existencia de un marco institucional en el que se han desarrollado las relaciones internacionales en el país.

En este sentido autores como Tickner (2007) señalan que acciones de intervención a gran escala en un país como Colombia han tenido lugar gracias a la existencia de lo que ha denominado un marco de política exterior de intervención por invitación; marco por medio del cual los gobiernos de Andrés Pastrana y Álvaro Uribe intensificaron la cooperación con Estado Unidos, y han solicitado la injerencia de ese país en los asuntos domésticos relacionados con la lucha antidrogas y contrainsurgente" (Tickner, 2007, pág. 91).

En este sentido, el Plan Colombia no debe ser entendido con un cambio brusco en las relaciones internacionales, es más podría considerarse que este Acuerdo de cooperación militar es la materialización del carácter de las relaciones internacionales del país. El cambio que se identifica en la primera etapa del surgimiento del Plan Colombia a finales de la década del noventa, es más de carácter cualitativo, según lo indica Tickner (2007), dicha transformación cualitativa estuvo dada por la nueva estrategia diplomática liderada por el presidente Andrés Pastrana, tanto en su etapa de campaña, como en los primeros años en el poder.

La estrategia diplomática se daba en términos de internacionalizar el conflicto armado colombiano, indicando que este no era sólo un problema de competencia nacional, sino que por su conexión con un delito de carácter transnacional como el narcotráfico este se constituía consecuentemente en una problemática de orden internacional, por lo cual los países más afectados con este delito debían actuar en colaboración con Colombia para contrarrestar los efectos adversos tanto del narcotráfico como del conflicto armado que permitía el fortalecimiento de los carteles, gracias a la alianza con los grupos insurgentes.

Ya con el Plan Colombia en marcha, y con la ruptura un par de años después del proceso de paz de san Vicente del Caguán se configuraba una nueva realidad, que vendría a afectar el panorama político interno, pero que a su vez el panorama político interno se vería afectado también por los cambios en la política exterior que se darían como consecuencia de los ataques terroristas del 11 de septiembre de 2001 en Estados Unidos.

\section{Guerra Internacional contra el terrorismo}

Con los ataques a Estados Unidos se iniciaría una nueva Era de las relaciones militares en el mundo, era en la cual la denominada Guerra Internacional contra el Terrorismo tendría el rol fundamental en el escenario mundial, y Colombia no fue la excepción, es más, casi que de forma inmediata el país en cabeza de Álvaro Uribe se convertiría en el principal aliado de EEUU en América Latina, asumiendo caso que de forma íntegra el discurso de Washington al respecto.

De modo que, si el rompimiento del proceso de paz serviría como plataforma política para que Álvaro Uribe ganara las elecciones presidenciales de junio de 2002, el nuevo discurso de Guerra Internacional contra el Terrorismo se constituiría en un bastión internacional que reafirmaría su 
discurso de enfrentamiento militar de los grupos insurgentes, en el cual se negaba la existencia de un conflicto armado interno, y se adoptaba la postura de la lucha contra el terrorismo al interior de Colombia.

En este sentido, durante el gobierno de Uribe se encontraría todo el respaldo del ejecutivo norteamericano en la lucha antiterrorista, por lo cual el Plan Colombia se vería fortalecido en su dinámica de cooperación militar, ya no sólo contra las drogas, sino que también contra los grupos insurgentes catalogados como terroristas y que ejercían un papel de aliados del narcotráfico en las regiones donde primaba el cultivo de alcaloides.

Respecto de los cambios en la configuración política de los Estados Unidos con el cambio de partido en el poder se debe señalar que, aunque en el entorno política hubo un cambio de un lado del espectro político a otro, se debe reconocer que respecto de la relación con Colombia, y la puesta en marcha del Acuerdo de Cooperación Militar se mantuvo el discurso contra las drogas ya predominante para la época.

Como se indica en Youngers (1997) la política antidrogas de Estados Unidos en el período de Bill Clinton (1993-2001) no sufrió mayores cambios, inclusive a pesar de las promesas de campaña en las que proponía dar un viraje a las relaciones, haciendo mayor énfasis en la demanda y no en la oferta, con una política antidrogas más cohercitiva que buscaba disminuir la demanda antes que realizar grandes inversiones con el fin de eliminar la oferta.

La guerra contra el terrorismo llevó a Estado Unidos a realizar cambios en su legislación de ayudas de cooperación internacional con el fin de poder establecer alianzas de manera más expedita para el combate a agrupaciones terroristas, estos cambios, de acuerdo a lo que señala Isacson, (2008): "un cambio en la ley de ayuda extranjera de Estados Unidos permitió a Colombia solicitar a ese país asistencia antinarcóticos para misiones no relacionadas con las drogas, como la lucha contra las guerrillas y los paramilitares” (Isacson , 2008 , pág. 2).

\section{Puesta en marcha del Plan}

El Plan Colombia se contempló inicialmente en un horizonte de tiempo de seis años hasta el 2005. En el primer año de vigencia del Plan el 35\% (DNP) del presupuesto del sector defensa de Colombia era el proveniente de los recursos del Plan Colombia, lo que muestra las proporciones de la ayuda, e injerencia de los Estado Unidos en el sistema de defensa del país durante la puesta en marcha del Plan.

Es importante reconocer los principales objetivos del Plan Colombia:

“i) Fortalecer las capacidades operacionales de la Fuerza Pública contra la cadena de producción del narcotráfico y la lucha contra los Grupos Armados al Margen de la Ley (GAML); ii) apalancar una estrategia de protección y asistencia social que permitiera fortalecer las condiciones económicas y sociales de los colombianos; iii) y coadyuvar a los procesos de Desarme, Desmovilización y Reinserción (DDR) junto con las reformas judiciales que estaban en curso en Colombia" (Departamento Nacional de Planeación, 2006, pág. 10).

De los objetivos que se planteaba el Plan se puede encontrar que existe una cobertura integrar en el combate antinarcóticos, donde el incremento de las capacidades del Estado y sus fuerzas militares constituía la piedra de toque para el fortalecimiento de la lucha y cerrar el cerco a los carteles de la droga y los grupos insurgentes vinculados como actores importantes de este negocio ilegal. 
En la evaluación realizada por el Departamento Nacional de Planeación en el año 2006 de la primera fase, se destaca que, más allá de la ayuda militar, el plan sirvió para dar pasos significativos en el mejoramiento y acceso tanto al sistema judicial, como a la protección y promoción de los Derechos Humanos (DD. HH.) Y el Derecho Internacional Humanitario (DIH).

De acuerdo con las cifras y los porcentajes de participación de cada uno de los componentes se puede encontrar que la mayor participación en el presupuesto del Plan recayó durante la primera fase en el componente de lucha contra las drogas ilícitas y el narcotráfico, con actividades como la fumigación con herbicidas y la erradicación de cultivos de coca por medio del método manual.

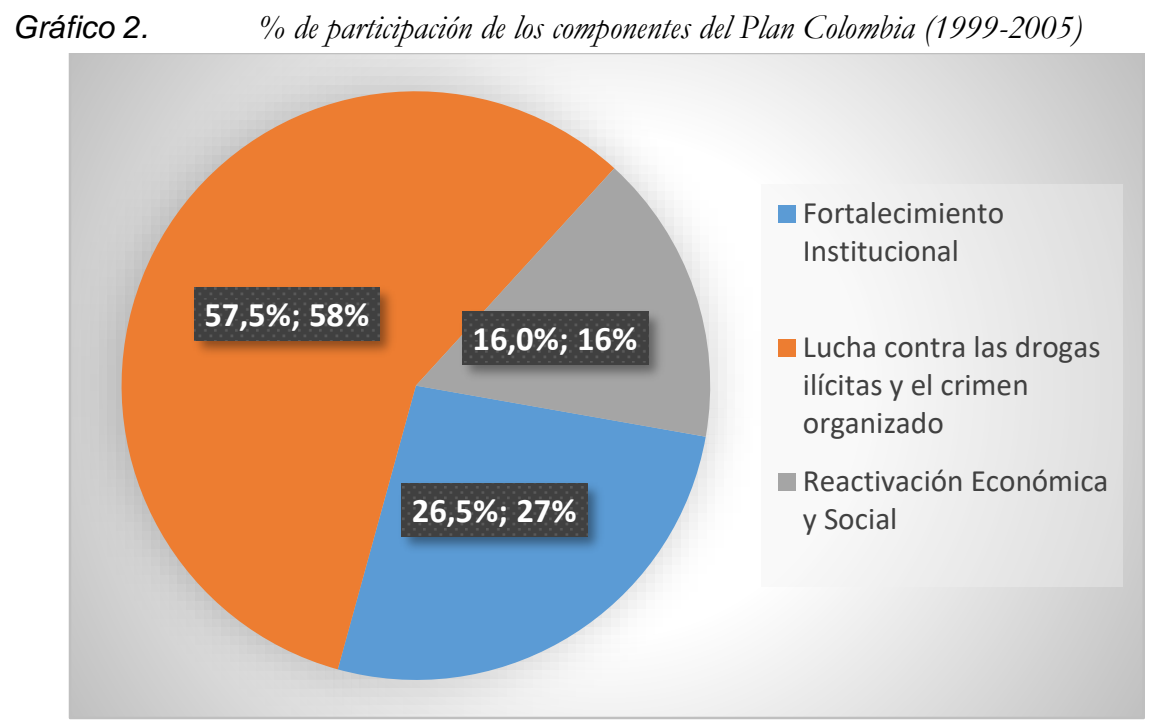

Fuente: Departamento Nacional de Planeación, 2016.

En este sentido, desde el punto de vista presupuestal puede afirmase que el principal énfasis del Plan Colombia, al menos durante la primera fase, estuvo concentrado en el combate al primer eslabón de la cadena del negocio del narcotráfico, es decir, el cultivo de drogas. Dejando quizá de lado a los principales componentes generadores de valor y desencadenantes de violencia como la transformación de la droga, el transporte y la comercialización.

Este énfasis en el combate de los cultivos ilícitos de drogas, y los argumentos que lo defendían se encontraban ya en los documentos del Departamento de Estado de los Estados Unidos denominado: International Narcotics Control Strategy Repor -informe publicado por el Departamento de Estado en el mes de marzo del año 2003-, transcurridos cerca de cuatro años de la puesta en marcha del Plan Colombia.Como se señala en Vargas Meza (2004) la estrategia antinarcoticos del país del norte se fundamenta en el combate a la oferta con el uso de la fuerza como mecanismo de disuación de los proveedores de droga.

Como se observa en el gráfico 2 los dos componentes que mayor proporción de recursos absorbieron del presupuesto del Plan Colombia en su primera fase son el de lucha contra las drogas y el crimen organizado y el fortalecimiento institucional que se realizaba a través de procesos de transferencias de capacidades tanto a las fuerzas militares como a las instituciones públicas de carácter civil. Finalmente, la distribución del presupuesto dejaba un lugar marginal para lo relacionado con el componente de reactivación económica y social donde se encontraban incluidas las acciones de apoyo 
al desarme y la reintegración, mediante el respaldo psicosocial y el apoyo para una efectiva integración a la vida económica con el apoyo de proyecto productivos que permitieran el sustento de los desmovilizados.

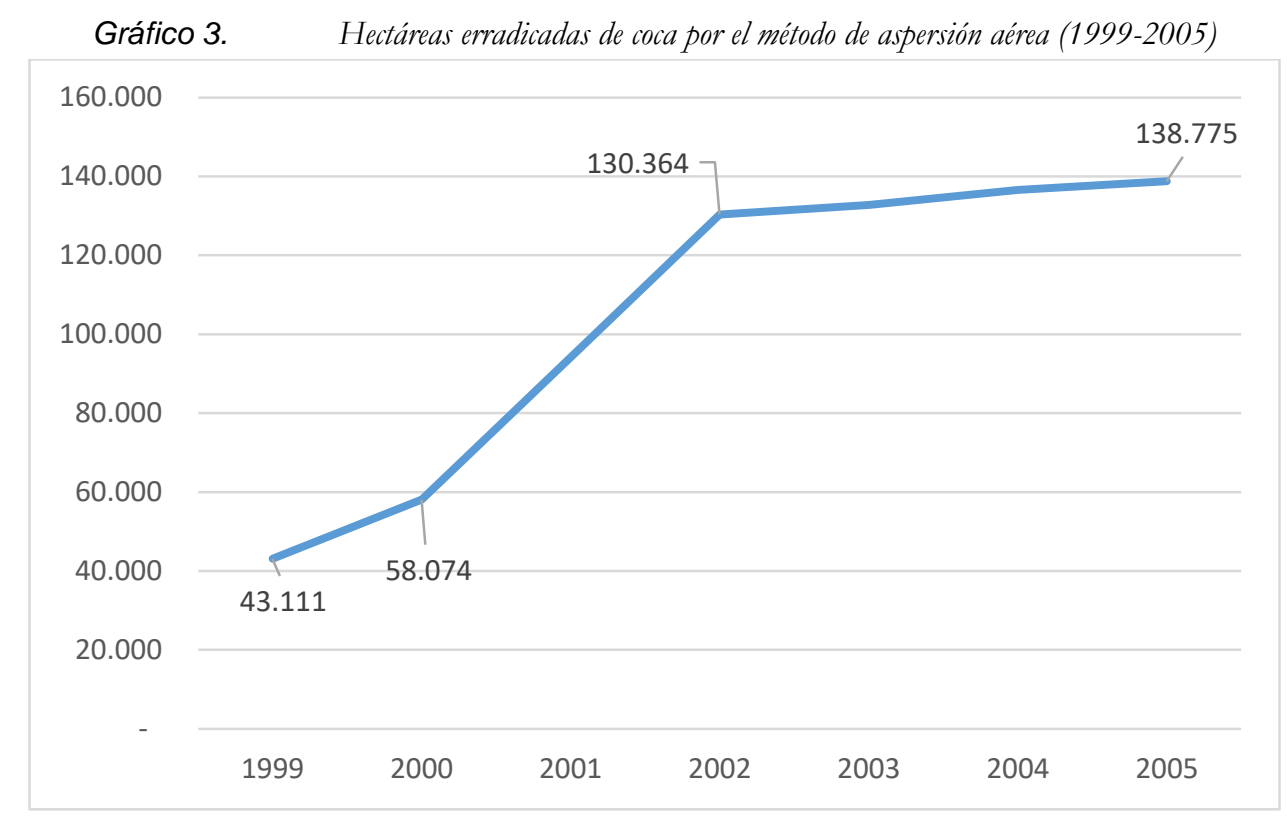

Fuente: Departamento Nacional de Planeación, 2006.

El mayor énfasis que se le dio a la erradicación de cultivos ilícitos como parte del componente de lucha contra las drogas permitió que en un lapso de tiempo de tres años, de 1999 a 2002, se lograra más que triplicar el número de hectáreas de coca asperjadas, como evidencia en el gráfico 3. Este incremento de la fumigación de cultivos ilícitos se extendería durante toda la fase 1, con una fumigación por año superior a las 130 mil hectáreas anuales mediante este método.

En la configuración de esta estrategia los contratistas norteamericanos encargados de llevar a cabo las labores de fumigación asumirían un papel preponderante en la configuración de actores de la lucha antidroga y el conflicto interno, en la medida que a cargo de ellos estaba el ejecutar en campo la principal acción estratégica del Plan Colombia durante la fase de inicio. Dichas acciones no estuvieron exentas de críticas (Tokatlan, sf), dado que durante toda la ejecución del Plan se acusó a las fumigaciones como provocadoras de contaminación ambiental y la posible generación de consecuencias adversas en la salud de las personas habitantes de las zonas afectadas por las fumigaciones masivas llevadas a cabo.

Estas críticas a las fumigaciones con glifosato llevaron a que el gobierno nacional solicitara en el año 2005 una serie de estudios científicos que determinaran los efectos sobre la salud que tenía la utilización de estos herbicidas en el marco del Plan Colombia (Solomon, Anadón, \& Cerdeira , 2005) (Vargas Meza, 1999). 
Gráfico 4. Hectáreas erradicadas de coca por el método manual (1999-2005)

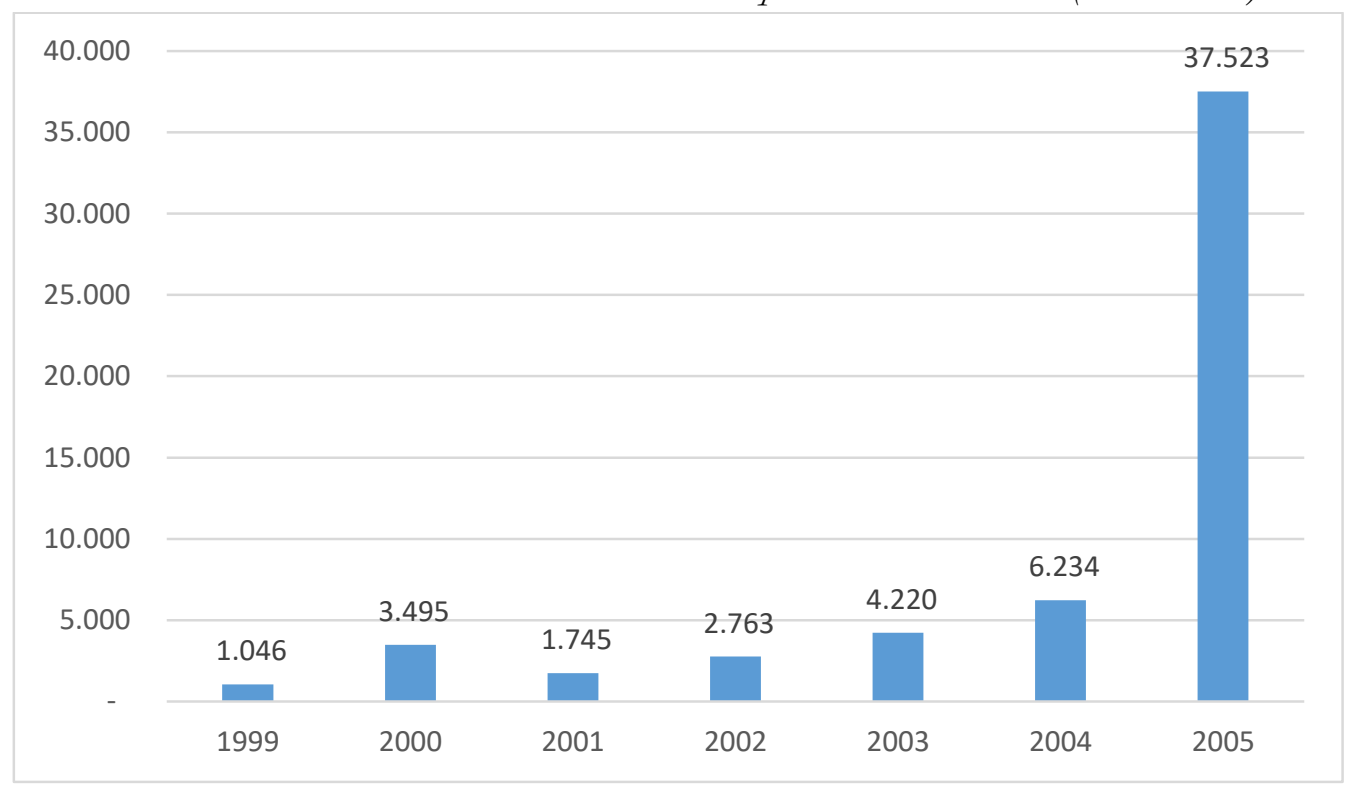

Fuente: Departamento Nacional de Planeación, 2006.

La revisión de la evolución de la erradicación manual de los cultivos de coca permite observar, tal y como se indica en la gráfica 4, que, si bien se presentó un aumento en el número de hectáreas erradicadas anualmente, éste no subió de una manera tan significativa como si lo hizo la erradicación por medio de fumigación aérea, sólo hasta el año 2005 se observa un aumento significativo del número de erradicaciones manuales.

Entendiendo los mayores riesgos asociados a la erradicación de cultivos de hoja de coca por medio de fumigación aérea, se esperaría un papel más activo de la erradicación manual, sin embargo, como de muestran las cifras, ha habido una preponderancia por la primera de las opciones, aun cuando la controversia sobre los costos y beneficios de dichas fumigaciones estuvo a la orden del día en esos primeros años.

En efecto, el mayor peso de la erradicación recayó desde el primer año en el método aéreo, como se muestra en el gráfico 5, donde por cada hectárea de cultivo de hoja de coca que se erradicaba manualmente se destruían 41 por el otro método. Se observa sin embargo que, en esta primera fase del Plan existe una tendencia a tener una razón de hectáreas que disminuía, lo cual en términos relativos significa mayor peso para la erradicación manual. 
Gráfico 5. No de Hectáreas erradicadas por el método de aspersión aérea por cada hectárea erradicada manualmente (1999-2005)

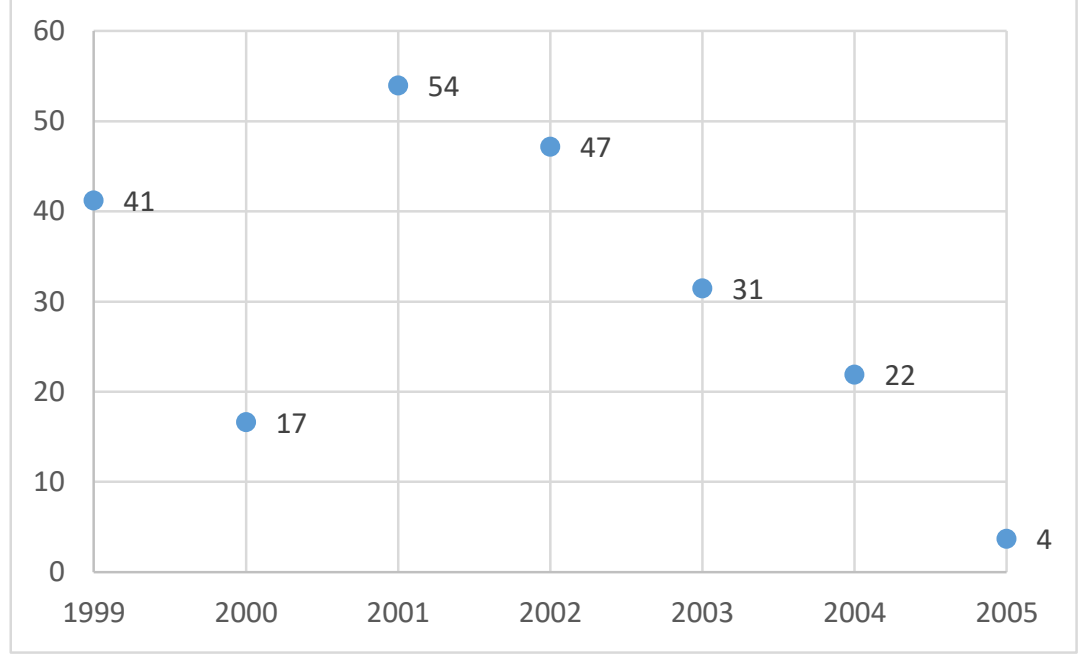

Fuente: DNP, 2006.

El nuevo enfoque centrado en el tráfico de droga y el reforzamiento militar, permitió al gobierno estadounidense intensificar su 'guerra contra la droga' y consolidar su acercamiento a los 'países proveedores’. (James Hawkins, 2009, pág. 106). Desde el momento de su firma oficial, el Plan Colombia ha ido más allá de un mero programa de las estructuras militares-policiales en Colombia sino que el mismo ha brindado la posibilidad a los Estados Unidos de tener una mayor injerencia militar en la región de Amérca Latina, particularmente en el conflicto interno colombiano. (James Hawkins, 2009, pág. 108)

De modo que, desde la perspectiva de los Estados Unidos el Plan va más allá de una mera estrategia bilateral con Colombia, sino que hace parte de una estrategia de un carácter internacional multilateral, así lo demuestra el hecho de a partir del 2002, todos los fondos estadounidenses que forman parte del Plan Colombia hayan sido cubiertos bajo el programa regional denominado Iniciativa Andina AntiDroga (IAA), lo cual es muestra de la visión regional en la cual el Plan Colombia encajaba como un instrumento más de acción.

\section{Segunda fase}

Desarrollada la primera fase del Plan Colombia el Gobierno de Álvaro Uribe Vélez encontró conveniente, como parte de su estrategia de lucha contra los grupos alzado en armas, continuar con la lucha antidroga como el bastión que permitiría un desenvolvimiento exitoso de la política de seguridad democrática.

En este sentido la extensión del Plan Colombia para una segunda fase se fundamentaba en la tesis de que los grupos armados ilegales del país permanecían en lucha contra el Estado por la existencia de una economía ilegal de drogas que los sostenía. Situación diferente a la que se presentaba en los países de América Latina que había enfrentado conflictos internos similares, pero que ante la ausencia de 
estructuras de financiación como la que bridaba el narcotráfico en Colombia no pudieron sostener su lucha insurgente durante mucho tiempo.

Desde el primer momento, la política de Seguridad Democrática encontró en el Plan Colombia su correlato a nivel internacional, lo que a su vez se cimentó en una política de ámbito internacional como la guerra internacional contra el terrorismo que sirvió de sustento al discursos de lucha contra las drogas como lucha contra el terrorismo, en la medida que había dos estrechos nexos entre estos dos delitos, básicamente, de acuerdo a como quedó plasmado en el Plan Nacional de Desarrollo 20022006, en los años previos al primer gobierno de Álvaro Uribe la violencia y los actos terroristas en el país habían aumentado significativamente, lo cual estaba estrechamente relacionado con el problema de las drogas (DNP, 2002).

Transcurridos los primeros años del período de gobierno 2002-2006 de Álvaro Uribe, se planteó la necesidad de ampliar más allá de lo contemplado inicialmente el Plan Colombia, en este sentido se presentó una apuesta denominada Estrategia para el Fortalecimiento de la Democracia y el Desarrollo Social, en la cual más allá de la lucha contra los cultivos ilícitos se contemplaba un importante componente social (Gómez M., 2007). Esta Estrategia del Gobierno Nacional quedó consignada en un documento del mismo nombre, el cual fue elaborado por Dirección de Cooperación Internacional de la Agencia Presidencia para la Acción Social y la Cooperación Internacional. (2007).

De acuerdo con lo planeado por el gobierno nacional de Colombia para la puesta en marcha de esta segunda fase del Plan eran necesarios recursos por un monto de 43.836,6 millones de dólares, con los cuales se tenía por objetivo principal consolidar, a través de una estrategia social, los avances que se lograron durante la fase uno.

Es importante señalar que esta segunda fase no contemplaba una reducción en el gasto militar en la lucha contra el tráfico de drogas. Sin embargo, como reconoce James Hawkins (2009), el plan hace especial énfasis en la inversión social, lo cual se argumenta bajo la tesis de que, para mantener el progreso en el país, se requiere el desarrollo y la presencia del gobierno en las zonas que han sido recuperadas en los últimos años. Con lo cual este mayor énfasis en los social estaría dirigido a incrementar la presencia del Estado con programas sociales en las zonas históricamente abandonadas.

Desde perspectivas como la de Bagley (2001), el enfasis principal en elcombate a la demanda indicaba que dificilmente el Plan Colombia tendría éxito,esto debido a que el fortalecimiento de las acciones militares, antes que acabar el negocio lo fortalecian en la medida que, como consecuencia no deseada por la estrategia antinarcóticos se provocaría un incremento delpoder armado de los narcotráficantes, que al sentirse atacado buscarían mayor poder armado para proteger sus negocios. Esto generaría espirales de violencia alrededor de la lucha armada contra los proveedores de droga.

De este modo la estrategia de lucha contra las drigas liderada por Estados Unidos, en la cual el principal objetivo lo constituyen los primeros eslabones del negocio, estrategia de encuentra su principal materialización en el Plan Colombia terminaría por convertirse en un actor más del conflicto por cuenta del mayor enfasis militar y la relación cada vez más estrecha entre actores armados con objetivos políticos y narcotráficantes.

\section{El Plan como nuevo actor del conflicto}


Sin duda que el Plan Colombia en sus primeros años de implementación generó un remezón en las relaciones de los actores armados y no armados en el conflicto armado interno, en este sentido es importante reconocer que el proceso de compatibilización del Plan con la política exterior y el contexto institucional colombiano fue lo que permitió a la postre que el Plan pudiera extenderse por 15 años.

Se puede señalar que, aunque la mayor expresión a través de la cual se hacía patente el Plan en el país, era por medio de los programas de fumigación, que como se señaló en la sección anterior no estuvieron exentos de polémica, en esa medida el Plan Colombia empezó a ser visto como un nuevo actor del conflicto, pues cada vez el narcotráfico y la lucha contra los grupos insurgentes se hacía más evidente ante los ojos de la opinión pública como un solo enemigo, producto de la simbiosis que se generaba tanto en el campo de batalla por la protección de los cultivos ilícitos, como en el discurso político que hacía todos los esfuerzos por presentarlo como un único enemigo.

Este juego que se empezó a establecer en el marco de este gran acuerdo de cooperación militar, ya no sólo en la erradicación de cultivos y las estrategias de fortalecimiento institucional, sino también en el juego político del conflicto interno, en la penetración de los discursos, entre otras actividades que fueron modificando la relación de los actores, en algunas ocasiones fortaleciéndolas y en otras resquebrajándolas, pero siempre generando una afectación en éstas.

En este sentido, y gracias a la preponderancia que adquirió el Plan a medida que se inició su implementación, es posible afirmar que la conjugación de cada uno de sus componentes permitió que el mismo se transforma en un eje de relacionamiento del conflicto armado, al punto de que no se podría entender el conflicto armado colombiano durante los primero años del siglo XXI de manera independiente al Plan Colombia, en la medida que éste se convirtió en uno de los temas centrales que desde las dos orillas de la confrontación se identificaba como punto de importancia para la superación del conflicto, ya sea que se viera el Plan como una oportunidad o un obstáculo para la superación de la guerra. Uno de los aspectos a tener en cuenta es que el Plan Colombia como tal era visto como un agente externo en algunas ocasiones debido a su carácter de acuerdo de cooperación internacional.

\section{Referencias bibliográficas}

Bagley, B. M. (2001). El tráfico de drogas y la política de Estados Unidos en Colombia. ICONOS, 87-99.

Departamento Nacional de Planeación. (2006). Balance Plan Colombia 1999-2005. Bogotá: DNP. Díaz Rivillas, B. (2002). Política exterior de los EE.UU. Hacia colombia: el paquete de ayuda de 1.300 millones de dólares de apoyo al plan colombia y la región andina. América Latina Hoy, 145-186.

Dirección de Cooperación Internacional de la Agencia Presidencia para la Acción Social y la Cooperación Internacional. (2007). Estrategia para el Fortalecimiento de la Democracia y el Desarrollo Social. Bogotá: Dirección de Cooperación Internacional de la Agencia Presidencia para la Acción Social y la Cooperación Internacional. Obtenido de www.mincit.gov.co/descargar.php?idFile $=221$

DNP. (2002). Plan Nacional de Desarrollo Hacia un Estado Comunitario (2002-2006). Bogotá: DNP. 
Gómez M., S. (1 de febrero de 2007). Plan Colombia fase II costa. El Tiempo. Obtenido de http://www.eltiempo.com/archivo/documento/MAM-2374995

Isacson, A. (2008). El Plan Colombia. Consecuencias no deseadas. Foreign Affairs En Español, 1-6.

James Hawkins, D. (septiembre de 2009). Reconfiguración del estado colombiano: el difícil balance entre consenso y coerción. Revista de Ciencias Sociales, 105-116.

Londoño, J. F. (2011). Colombia y Estados Unidos: una relación por revisar. En D. Cardona Cardona, Colombia: una política exterior en transición. (págs. 235-272). Bogotá-Colombia: Fundación Friedrich Ebert en Colombia (Fescol).

Mendez, M. (1976). Panamá, el Canal y la Zona del Canal. NUEV A SOCIED AD, 125-140.

Solomon, K., Anadón, A., \& Cerdeira , A. (2005). Estudio de los efectos del Programa de Erradicación de Cultivos Ilícitos mediante la aspersión aérea con el herbicida Glifosato (PECIG) y de los cultivos ilícitos en la salud bumana y en el medio ambiente. Washington, D.C.: Comisión Interamericana para el Control del Abuso de Drogas (CICAD).

Tokatlan, J. G. (sf). Estados Unidos y los cultivos ilícitos en Colombia : los trágicos equivocos de una fumigación futil. Obtenido de www.academia.edu: http://www.academia.edu/download/34620026/working_paper_tokatlian.doc

Vargas Meza, R. (1999). Fumigación y conflicto: politicas antidrogas y deslegitimación del Estado en Colombia. Bogotá: Tercer Mundo.

Vargas Meza, R. (2004). Drogas, conflicto armado y seguridad global en Colombia. Nueva sociedad, 117-131.

Vega Cantor, R. (2012). Colombia en la geopolitica hoy. El Ágora USB, 367-402. doi:http://dx.doi.org/10.21500/16578031.81

Youngers, C. (1997). La guerra antidrogas: Estados Unidos sigue un camino equivocado. Obtenido de Transnational Institute: https://www.tni.org/en/article/stub-271 